\title{
In situ Method for Power Re-Equalization of Wavelength Pulses Inside of OCDMA Codes
}

\author{
Tolulope B. Osadola, Student Member IEEE, Siti K. Idris, Ivan Glesk, Senior Member, IEEE, \\ Kensuke Sasaki, and Gyaneshwar C. Gupta
}

\begin{abstract}
A simple in-situ method to equalize power among individual wavelengths pulses representing two-dimensional wavelength-hopping time-spreading OCDMA code originally generated by a fibre Bragg grating-based OCDMA encoder is presented. Experimental data obtained in a field-based multiuser OCDMA testbed shows that applying this method results in system performance enhancements which was demonstrated by observing improved bit error rate (BER) during the field trials.
\end{abstract}

Index Terms-Fiber Bragg Grating, Optical CDMA, Wavelength-Hopping Time-Spreading codes, OCDMA testbed.

\section{INTRODUCTION}

$\mathrm{O}^{\prime}$ PTICAL code division multiple access (OCDMA) is one possible family of optical multiple access techniques for the next generation broadband networking which has attracted considerable attention in the past decade [1] - [7], [17], [19]. Presently, the prevailing multiple access technologies used in optical fibre networks are the electrical time division multiple access (TDMA) in which the transmission medium is shared by users based on each user transmitting in its own allocated timeslot [1] and wavelength division multiplexing (WDM) [2] which enables users to simultaneously transmit data by making use of different wavelength channels. OCDMA extends the benefits of both TDMA and WDM by adding an additional level of data privacy at the physical layer by identifying user information on the network based on the uniquely assigned optical codes. The implication of this is that apart from receiver with previous knowledge of the user's transmitted code which is needed to decode the transmitted coded information, the same information is seen as noise called multi access interference (MAI) by other users without such prior knowledge. In the light of this, it is important that codes for OCDMA systems are designed in a way that will

Manuscript received February 16, 2011. This work was supported in part by the GRPe grant.

Tolulope B. Osadola, Siti K. Idris, and Ivan Glesk are with The Center for Intelligent and Dynamic Communications, Electronic and Electrical Engineering Department, University of Strathclyde Glasgow. G1 1XW, UK. (Phone: +447403034903; e-mail: tolulope.osadola@strath.ac.uk, siti.idrisothman@strath.ac.uk, ivan.glesk@eee.strath.ac.uk.

Kensuke Sasaki, and Gyaneshwar C. Gupta., are with Oki Electric Industry, Warabi-shi, Saitama, Japan. (E-mail: sasaki356@ oki.com, gupta852 @ oki.com). preserve its uniqueness regardless of the constraints or imperfections of the optical network components and transmission media. Examples of incoherent OCDMA codes include various forms of optical orthogonal codes (OOC) [3], [4] and various families of prime codes [5], [6]. An important family of two dimensional OOC formed using both wavelength and time dimensions is the wavelength hopping time spreading (WH/TS) family of codes [7]. Various authors have studied different aspects relevant to the successful implementation of OCDMA [8], [9] in anticipation of recording tremendous success similar to wireless CDMA. Also of interest has been research into all optical means of signal processing [17], [18] with the aim to further improve OCDMA system performance. It is expected that optical processing can help to overcome the limitation imposed by the bandwidth limited electronic signal processing. Optical encoding/decoding which refers to the process of assigning OCDMA codes to user information at the transmitter side and intelligently extracting this information from coded signals in the presence of MAI at the receiver side, is an important aspect of optical signal processing. Various encoding and decoding methods exploring different technologies and approaches exists and were demonstrated for use with incoherent two dimensional OCDMA encoders and decoders. These include the use of thin film filters in combination with optical delay lines [10], holographic Bragg gratings [11], and fibre Bragg gratings [12]. Of the above mentioned methods, it is worthy of noting that fibre Bragg gratings while inexpensive, provide very promising approach towards miniaturisation and complexity as the function of wavelength filtering is combined with desired time delays in one single fiber device. Fibre Bragg gratings (FBGs) have been widely used in optical communications signal processing for some time [12].

The use of FBGs for encoding and decoding of WH/TS optical codes in OCDMA systems by spreading multiple wavelengths within a bit period and placing them into time chips at the transmitter end and reciprocally de-spreading them and forming an autocorrelation peak at the receiver end is a widely known technique [12], [13]. However, once signal is encoded and OCDMA code had been generated by the FBG encoder, the individual wavelengths of the WH/TS OCDMA 
code cannot be any more easily individually accessed for a selective processing if that is what is desired. In current approaches these individual wavelengths will have to be first accessed by demultiplexing the multiwavelength OCDMA code then individually attenuated for the proper power equalization and then multiplexed back while preserving the original time spreading. All the above adds additional hardware complexity and also increases the overall system cost.

In this paper we present to the best of our knowledge for a first time a simple in-situ method allowing power redistribution among individual wavelength pulses within the WH/TS OCDMA code after such code generation by the FBG encoder. Experimental data obtained from our trials using field-based multiuser incoherent OCDMA testbed showed that by applying proposed in-situ method significant system performance improvements judged by obtained bit error rates (BER) can be achieved.

\section{BACKGROUND}

The effect of signal polarization on a single mode fiber transmission link with added section of multimode fiber was noted in [14]. It was shown that manipulating the signal polarization before launching into a single mode fibre via a section of multimode fiber can result in system performance changes. However, this study was only limited to a single wavelength being transmitted. In our quest to improve OCDMA system performance we explored and expanded the above observations to OCDMA multi wavelengths systems based on two dimensional wavelengths hopping time spreading codes for data communication. We found that introducing the combination of a polarization controller and a short length of multimode fiber (PLC-MMF) right before the OCDMA coded signal is presented to the OCDMA decoder will result in the dramatically improved OCDMA system performance judged by the BER.

It is well known that in the presence of multiple access interference, the data detection by the OCDMA receiver will depend on many factors. In OCDMA systems with coding schemes based on multi-wavelength pulses, in order to achieve the best performance of the OCDMA system, the theory prescribes [5] that these individual wavelengths pulses within the generated OCDMA code should have equal power levels. Fulfilling this requirement is essential towards achieving the desired power relationship between auto and cross-correlation signals in the presence of MAI. This is because during the signal reception the OCDMA receiver/decoder produces besides the autocorrelation peak carrying data also undesired cross correlations as the result of seeing and incorrectly decoding transmitted codes from simultaneous network users. It is obvious that depending on relative positions among individual wavelengths pulses within these individual WH/TS codes, there will be always a non-zero probability that some of these wavelength pulses during decoding process will "accidentally" overlap in such a manner which will result in cross-correlations of similar or even taller heights than is the desired correlation peak itself [16]. This will then cause a significant data corruption resulting in undesirable degradation of bit error rate (BER).

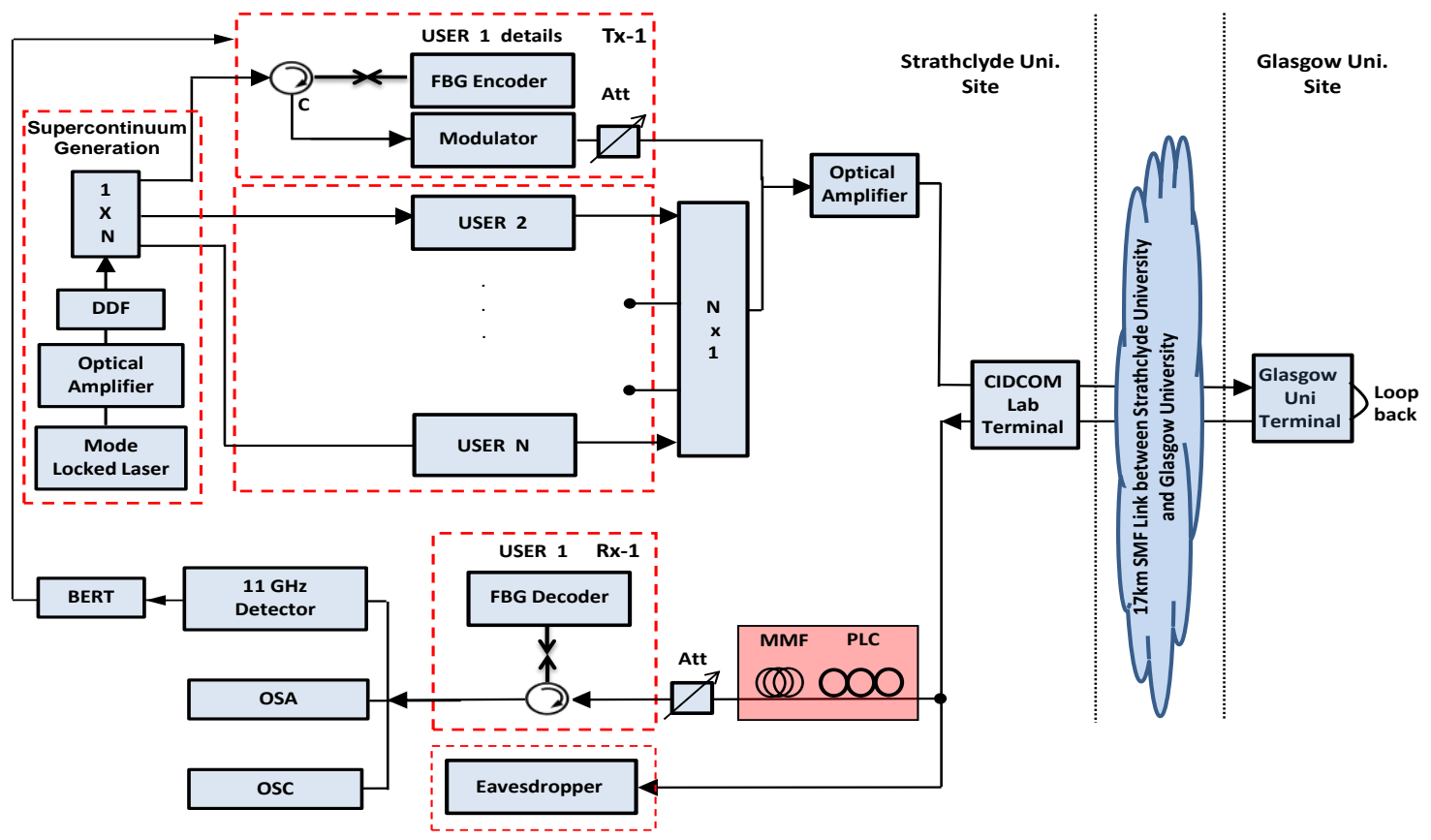

Fig. 1. Experimental setup to demonstrate wavelength power redistribution in OCDMA transmission using PLC-MMF. C-Circulator, Att-Attenuator, OSAOptical Spectrum Analyser, OSC-Oscilloscope, DDF-Dispersion decreasing fiber. 


\section{EXPERIMENTAL DEMONSTRATION}

To demonstrate the effectiveness of the proposed in situ technique leading to system performance improvements, the proof of concept experiments were carried out using a field based fiber optic transmission link having a length of $17 \mathrm{~km}$. The transmission link is fully compensated for the chromatic dispersion and at its both ends is connected to multiuser OCDMA terminals, see Fig. 1. Each user's data is uniquely encoded using FBG-based encoders with WH/TS OCDMA codes. Transmitted power level for each user was equalized by the variable optical attenuator at the output of each encoder. USER 1 encoder is matched with the corresponding decoder at the receiving end. The testbed during our experiments was populated with four simultaneously transmitting OCDMA users and two receivers. The combined traffic when presented to OCDMA decoder matched to USER 1's encoder will produce weight four autocorrelation peak corresponding to decoded transmission from USER 1, while all unmatched codes coming from simultaneous users broadcasting on the network will result in cross correlations thus contributing to the so called multiple access interference, MAI. The OCDMA testbed uses $2 \mathrm{D}$ - $(4,16)$ carrier-hopping time-spreading prime codes which consist of four wavelengths $\left(\lambda_{1}=1551.72 \mathrm{~nm}, \lambda_{2}=1550.92 \mathrm{~nm}, \lambda_{3}=1552.52 \mathrm{~nm}, \lambda_{4}=\right.$ $1550.12 \mathrm{~nm}$ ) based on $100 \mathrm{GHz}$ ITU grid positioned in 16 time chips each of 25ps duration. Each code comprises of all four wavelengths. Individual wavelengths are generated by spectral slicing of a $3.2 \mathrm{~nm}$ wide optical supercontinuum (OSC) resulting from pulse compression of 1.8ps FWHM laser pulse from an Erbium doped mode locked fiber laser (MLL) running at OC-48. To generate OSC, the laser pulse is passed through an erbium doped fiber amplifier followed by the dispersion decreasing fiber. The optical supercontinuum needed by testbed users for OCDMA codes generation is obtained by its power splitting (see fig. 1). Each portion is immediately encoded by user's own FBG-based OCDMA encoder. This produces WH/TS OCDMA codes. As shown in details for USER 1 codes are then modulated by Mach-Zehnder data modulator driven by a $2^{31}-1$ PRBS data from an Agilent N4903A series bit error rate tester. The coded sequences from remaining encoders (USER 2-4) are first combined with the USER 1 traffic, amplified and then launched into a $17 \mathrm{~km}$ dispersion compensated field fiber link. For the purpose of these experiments, the received signal before it gets decoded was launched into a module containing a polarization controller and a short $(60 \mathrm{~cm})$ section of multimode fiber (see PLC-MMF in fig. 1) and thereafter was presented to USER 1 FBG-based OCDMA decoder. Since the FBG decoder provides reverse wavelength delays compare to the encoder, this result in the strong autocorrelation peak of weight four at the background of cross-correlations. After OCDMA decoding, the decoded signal was sent via an optical power meter with build in attenuator (Agilent 8157A) to a bit error rate tester with an $11 \mathrm{GHz}$ optical receiver (Nortel PP10G) as its frontend. The decoded OCDMA signal was also monitored using an optical spectrum analyzer (Agilent 86146B) and an oscilloscope (Digital communications analyzer - Agilent $86105 \mathrm{~B}$ equipped with $60 \mathrm{GHz}$ optical sampling head).
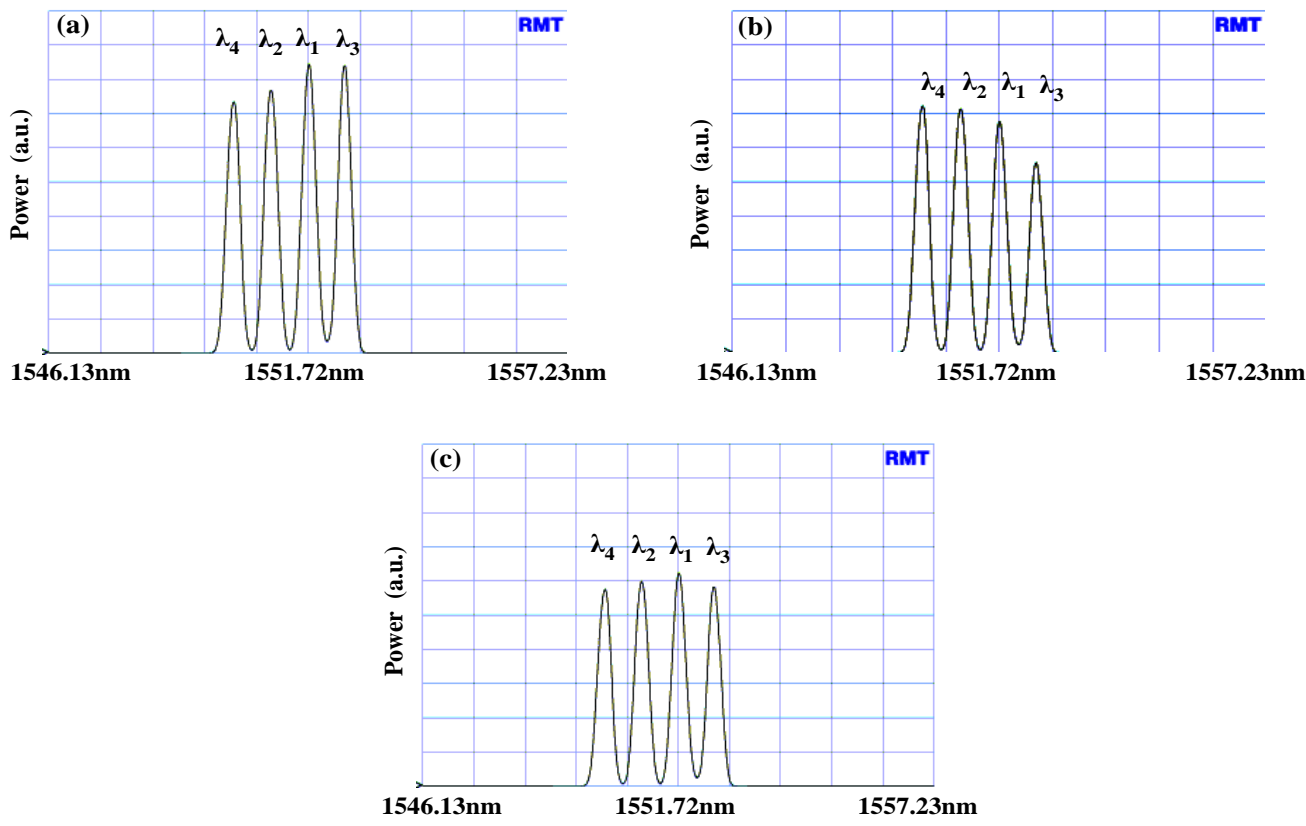

Fig. 2. Optical spectrum of OCDMA signal showing the power distribution among individual wavelengths within the code: (a) at the input of PLC-MMF module, (b) and (c) after passing PLC-MMF module with different settings of PLC, respectively. 
To study the effectives of the proposed in situ technique to control and change the wavelengths power distribution within OCDMA codes during the transmission while inside of the optical fiber link, a module containing a generic polarization controller from FiberControl followed by a short $(60 \mathrm{~cm})$ piece of multi mode fibre was inserted right before the FBG-based OCDMA decoder belonging to USER 1 (see Fig. 1). At this point the signal was already affected by its propagation in the $17 \mathrm{~km}$ long single mode fiber link outside the laboratory. The link is part of the advanced bidirectional fiber optic OCDMA testbed connecting CIDCOM research laboratory at University of Strathclyde with the Communication laboratory at Glasgow University. Numerous experiments were conducted to study and verify the effectives of the proposed in situ technique to control and change the wavelengths power distribution within OCDMA codes while they are still inside of the optical fiber link. Some examples from obtained results are shown in Fig. 2. Figure 2(a) shows optical power spectrum of the received 2D-WH/TS OCDMA code showing an uneven distribution of power among individual wavelengths within the OCDMA code. Figures 2(b) and 2(c) demonstrate that considerable changes of such wavelengths power spectrum are achievable by using the proposed in situ technique by simply manipulating signal polarization via the polarization controller inside the MMF-PLC module. During these experiments we also found that regardless of the "undesired influence" of the transmission fiber link on the propagated signal (OCDMA codes) we were always able to change the optical power spectrum (wavelength power distribution) of the OCDMA code by manipulating the incoming signal polarization just before the coded sequences were presented to the OCDMA decoder at the receiver end.

Our second set of experiments was designed to verify if the ability to control the optical power spectrum of the OCDMA code can be used for improving the overall OCDMA system performance, in particular, the bit error rate observed by OCDMA users. For the purpose of this demonstration we picked the USER 1.

All measurements were taken at the presence of MAI caused by three additional simultaneous users broadcasting on the network. To conduct these experiments we again used the $17 \mathrm{~km}$ long bidirectional optical fiber link connecting the CIDCOM research laboratory at University of Strathclyde with the Communication research laboratory at Glasgow University. For an illustration Fig. 3(a) is Tx-1 output showing the waveform of OCDMA code 1 modulated with USER 1 data as seen on the oscilloscope via an $11 \mathrm{GHz}$ bandwidth limited optical receiver. It is worthwhile to note that because of an insufficient time resolution $\lambda_{1}, \lambda_{4}$ and $\lambda_{2}, \lambda_{3}$ wavelength peaks within the code 1 are not properly time resolved. Figure 3(b) shows the autocorrelation resulting from OCDMA data decoding by USER 1 OCDMA decoder in the absence of multiple access interference, MAI (USERs 2, 3, and 4 are "OFF" and not broadcasting on the network). Figure 3(c) and 3(d) shows the decoded OCDMA signal by the USER 1 OCDMA decoder when all users are "ON". In this case the decoder output is the combination of an autocorrelation corresponding to the received USER 1 data and cross correlations (MAI) resulting from simultaneous users (USER 2-4) also broadcasting on the network. By comparing Fig. 3(c) and 3(d) we can notice that the contrast ratio between the autocorrelation peak and the "nearby" MAI is lower in Fig. 3(c) than it is in Fig. 3(d) where the PLC-MMF module was implemented. This observed change will have impact on the overall system performance. It will also affect the BER.

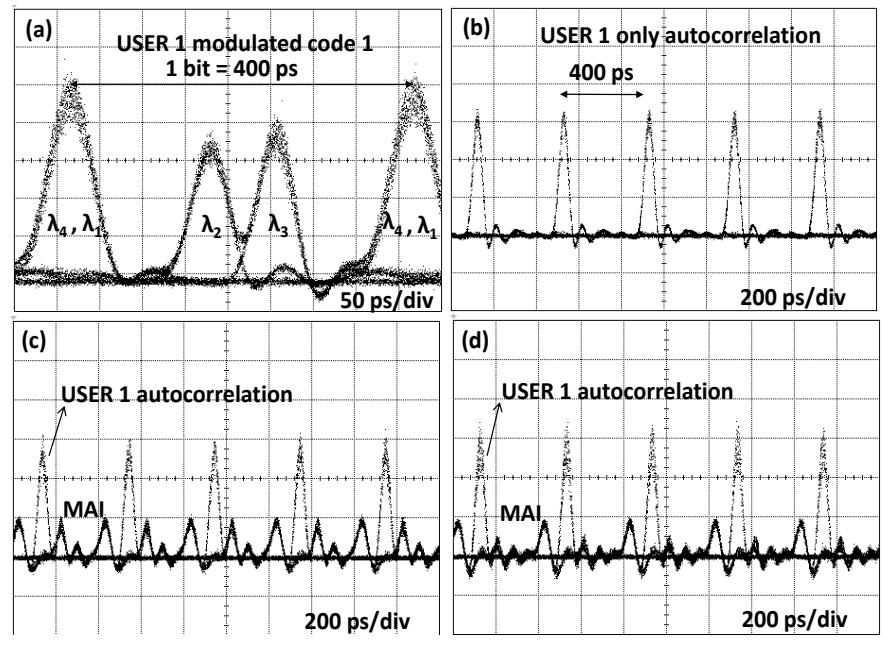

Fig. 3(a). OCDMA code 1 modulated with USER 1 data as seen on the oscilloscope via an $11 \mathrm{GHz}$ bandwidth limited optical receiver, (b) obtained autocorrelation after decoding a single user OCDMA data transmission by USER 1, (c) and (d) signal at the USER 1 OCDMA decoder output showing autocorrelation peaks corresponding to received USER 1 data and the MAI resulting from simultaneous users broadcasting on the network without and with PLC-MMF module implementation, respectively.

Obtained BER results are summarized in Fig. 4 which shows two sets of data measurements:

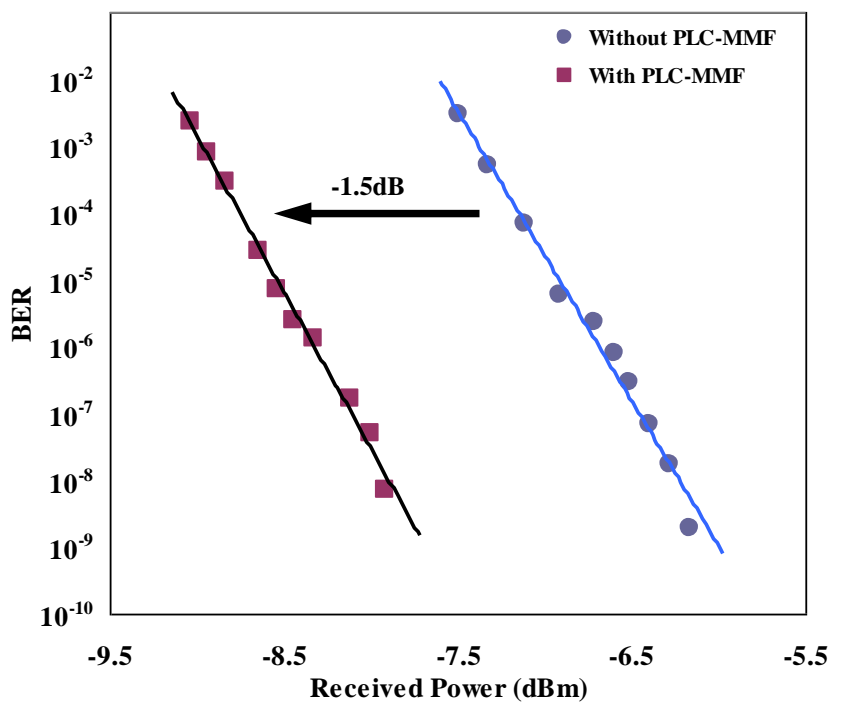

Fig. 4. BER versus received optical power for case of OCDMA transmission over $17 \mathrm{~km}$ long bidirectional fiber optical link between Strathclyde and Glasgow University. Squares: PLC-MMF module used; circles: no PLCMMF module used.

The first set of measurements (see squares in Fig. 4) was performed with inserted PLC-MMF module as can be seen in 
Fig. 1. Then the bit error rate was recorded for varying power levels. Prior to recordings the PLC-MMF module was used to target the most even wavelength power distribution in the OCDMA code (see example Fig. 2(c) and Fig. 3(c)). This was monitored by optical spectrum analyzer and oscilloscope. We found that once the proper polarization setting was found it was not necessary to make additional adjustments during subsequent measurements.

The second set of measurements (see circles in Fig. 4) was performed using the same setup but without the PLC-MMF module being inserted.

Comparison of results obtained from both sets of measurements in the form of BER curves can be seen in Fig. 4. The results clearly indicate a $1.5 \mathrm{~dB}$ system performance improvement (marked as $-1.5 \mathrm{~dB}$ power penalty) when the PLC-MMF module was used.

\section{DISCUSSION}

We have demonstrated a simple novel in situ method for reequalising the power distribution of individual wavelength pulses representing two-dimensional wavelength-hopping time-spreading (2D-WH/TS) OCDMA codes. The method is based on manipulating the polarisation of incoming signal by using a module combining a polarisation controller followed by a short section $(\sim 60 \mathrm{~cm})$ of multimode fiber inserted right before the OCDMA decoder. We have shown that invoked polarization changes via the PLC-MMF module will affect the power distribution among individual wavelengths pulses of the 2D-WH/TS OCDMA code. To confirm the effectives of these observations, field trials were conducted using $17 \mathrm{~km}$ long multiuser OCDMA testbed based on a bidirectional single mode fiber optic transmission link compensated for chromatic dispersion with subpicosecond accuracy installed between the CIDCOM laboratory at University of Strathclyde and the Communication laboratory at Glasgow University. These experiments confirmed that proposed in situ method provides very effective way to control and equalize the power spectrum of 2D-WH/TS OCDMA codes while propagating in the single mode optical fiber with no need for accessing the code's individual wavelengths to perform such power readjustment if and when necessary.

We also confirmed experimentally that by applying the in situ method on the incoming OCDMA signal right before it is presented to the 2D-WH/TS OCDMA decoder the BER system performance improvement can be achieved. We observed negative $1.5 \mathrm{~dB}$ power "penalty" resulting in the left shift of the BER curve as indicated in Fig. 4.

\section{CONCLUSION}

A simple novel in situ method to re-adjust the optical power spectrum of two-dimensional wavelength-hopping timespreading OCDMA codes while propagating in the optical fiber link was developed and demonstrated. To achieve such readjustment there is no need to access any wavelength individually. When applying the in situ method on an incoherent OCDMA system using 2D-WH/TS OCDMA with four simultaneous users the receiver BER performance was improved by $1.5 \mathrm{~dB}$. All experiments were conducted using $17 \mathrm{~km}$ long fiber link connecting University of Strathclyde with University of Glasgow showed.

\section{REFERENCES}

[1] P. R. Prucnal, I. Glesk, P. Toliver, R. Runser, and K. L. Deng, "Signal processing in high speed OTDM networks," in Proc. 24th European Conf. Optical Communication, vol.3, pp. 5-19, 20-24 Sep. 1998.

[2] J. Berthold, "Evolution of WDM in transport networks," Optical Fiber Communication Conference and Exhibit, OFC '98, Technical Digest, pp. 133, 22-27, Feb. 1998.

[3] H. Chung and P. Kumar, "Optical orthogonal codes - new bounds and optimal construction," IEEE Trans. on Info. Theory, vol. 36, no. 4, pp. $886-873,1990$

[4] N. Tarhuni, "Multiclass optical orthogonal codes for multiservice optical CDMA networks," J. Lightwave Technol., vol. 24, no. 2, pp. 694-704, 2005.

[5] G.-C. Yang and W. C. Kwong, Prime codes with applications to cdma optical and wireless networks, Boston: Artech House, 2002.

[6] Z. Wei and H. Ghafouri-Shiraz, "Proposal of a novel code for spectral amplitude coding optical CDMA systems," IEEE Photonics Tech. Letters, vol. 14, no. 3, pp. 414-416, 2002.

[7] L. Tancevski and I. Andonovic, "Wavelength hopping time spreading code-division multiple-access systems," Elect. Lett. vol. 30, pp. 13881390, 1994,

[8] N. Kostinski, K. Kravtsov, and P. R. Prucnal, "Demonstration of an alloptical OCDMA encryption and decryption system with variable twocode keying," IEEE Photonics Technology Letters, vol. 20, no. 24, pp. 2045 - 2047, Dec. 2008.

[9] I. Glesk, P. R. Prucnal, and I. Andonovic, "Incoherent ultrafast ocdma receiver design with $2 \mathrm{ps}$ all-optical time gate to suppress multiple-access interference, "IEEE Journal of Selected Topics in Quantum Electronics, vol.14, no.3, pp. 861-867, May-June 2008

[10] I. Glesk, V. Baby, C.-S. Brès, Y.-K. Huang, and P. R. Prucnal, "Performance Enhancement of Optical CDMA Systems Using Ultrafast All-Optical Sampling," In proceedings IEEE-LEOS 2005 Avionics Fiber-Optics Conference, Minneapolis, MN, 20-22 Sept. 2005, paper ThA1, pp. 58-59.

[11] Y. K. Huang, K. Kravtsov, I. Glesk, P. R. Prucnal, C. M. Greiner, D. Iazikov, and T. W. Mossberg, "Integration of dual-code optical CDMA encoder and decoder by holographic Bragg reflectors, "Optical Fiber Communication and the National Fiber Optic Engineers Conference, 2007. OFC/NFOEC 2007 pp.1-3, 25-29 March 2007.

[12] Y. Zhang, H. Chen, Z. Si; H. Ji, and S. Xie, "Design of FBG en/decoders in coherent $2 \mathrm{~d}$ time-wavelength OCDMA systems, "Photonics Technology Letters, IEEE, vol.20, no.11, pp .891-893, June 2008.

[13] A. Grunnet-Jepsen, A. E. Johnson, E. S. Maniloff, T._W. Mossberg, M. J. Munroe, and J. N. Sweetser, "Fibre Bragg grating based spectral encoder/decoder for lightwave CDMA," Electronics Letters, vol. 35, no.13, pp. 1096-1097, 24 June 1999.

[14] S. S.-H. Yam, F.-T. An, M. E. Marhic, and L. G. Kazovsky, "Polarization sensitivity of $40 \mathrm{~Gb} / \mathrm{s}$ transmission over short-reach $62.5 \mu \mathrm{m}$ multimode fiber using single-mode transceivers," Optical Fiber Communication Conference, 2004. OFC 2004, vol. 2, pp. 3, 23-27 Feb. 2004.

[15] W. C. Kwong, P. A. Perrier, and P. R. Prucnal, "Performance comparison of asynchronous and synchronous code-division multipleaccess techniques for fiber-optic local area networks," IEEE Trans. Comm., vol. 39, no. 11, pp.1625-1634, 1991.

[16] C. Michie, R. Atkinson, I. Andonovic, I. Glesk, P. R. Prucnal; K. Sasaki, and G. Gupta, "Interferometric Noise Characterisation of a 2-D Time spreading Wavelength Hopping OCDMA Networks using FBG Encoding/Decoding," ICTON '07, vol. 1, pp. 114-117, 1-5 July 2007.

[17] K. I. Kitayama, X. Wang, and N. Wada, "OCDMA over WDM PON solution path to gigabit-symmetric FTTH," J. Lightwave Technol., vol. 24, no. 4, pp. 1654-1662, Apr. 2006.

[18] Y. K Huang, B. Wu, I. Glesk, K. Kravtsov, E. Narimanov, P. R. Prucnal, and T. Wang, "Achieving Physical Layer Security/Privacy with Selfwrapped OCDM transmission," MILCOM 2007, pp. 1-5, 29-31 Oct. 2007.

[19] N. Minato, H. Tamai, H. Iwamura, S. Kutsuzawa, S. Kobayashi, K. Sasaki, and A. Nishiki, "Demonstration of 10Gbit/s-Based TimeSpreading and Wavelength-Hopping Optical-Code-Division- 
Multiplexing Using Fiber-Bragg-Grating En/Decoder", IEICE Trans. Comm., vol. E88-B, no.10, pp. 3848-3854, Oct. 2005.

Tolulope Osadola (S'09) received the M.Sc. degree in Communications Control and Digital signal processing (with distinction) in 2009 from the University of Strathclyde in Glasgow where he is currently studying for his $\mathrm{PhD}$ degree in Electronic and Electrical engineering at the Center for Intelligent and Dynamic Communication(CIDCOM).

His area of current research interests includes ultra short pulsed laser, ultrafast all-optical data processing, and optical network security

Tolulope Osadola is a recipient of the Scottish overseas research students' award and the University of Strathclyde postgraduate research studentship.

Siti Idris received her M.Sc. from Microelectronic at Universiti Kebangsaan Malaysia (UKM). In 2002 she joined Faculty of Electronic and Computer Engineering, Universiti Teknikal Malaysia Melaka (UTeM) as a lecturer. As the recipient of Malaysian Government Sponsorship she is pursuing her $\mathrm{PhD}$ at University of Strathclyde, Glasgow. Her research interests are ultrafast alloptical data processing and switching.

Ivan Glesk (M'01-SM'01) received the Ph.D. degree in quantum electronics and optics from Comenius University, Bratislava, Slovak Republic, in 1989 and the D.Sc. degree from the Slovak Academy of Sciences, Bratislava. In 1986, he joined the Department of Experimental Physics, Comenius University, where he later became a Professor of physics, and was engaged in research in the areas of nonlinear optics, laser physics, and light detection and ranging (LIDAR) methods of remote sensing the atmosphere. In 1991, he joined Princeton University, USA were he was Manager of the Lightwave Communication Research Laboratory and Senior Research Scholar in the Department of Electrical Engineering. In 2007, he joined University of Strathclyde, Glasgow, U.K., as a Professor of Broadband Communication Systems in the Department of Electronic and Electrical Engineering. His current research interests include applications of ultra short pulsed laser systems for optical networks, ultrafast all-optical signal processing, and optical interconnects. He is author or coauthor of more than 280 publications, 16 book chapters and holds five patents. He is on the Editorial Board of the International Journal of Optics and Optica Aplicata and is a recipient of the International Research and Exchanges Board (IREX) Fellowship.

Kensuke Sasaki received the B.S. degree in physics from Shinshu University, Nagano, Japan in 1984 and Master degree in material science from Osaka Prefecture University, Japan in 1987. He joined Oki Electric Industry Co., Ltd., Tokyo, Japan in 1987. He has developed an electro-absorption modulator and Fiber Bragg Grating devices. He is currently working at Corporate Research \& Development center. His current research interest focuses on developing Fiber Bragg Grating based en/decoders for OCDM system for the next generation access network.

Gyaneshwar Chandra Gupta is with Oki Corporate Research \& Development Center, Tokyo Japan where he is involves in next generation broadband access networks, high-speed opto-electronics devices and high power amplifiers for $3 \mathrm{G}$ and WiMax wireless base station. Dr. Gupta has over 17 years of international experience working prestigious research institutions across the globe, including Optel (Fujitsu India), NEC Center Research Labs, Japan, Lucent Technologies/Bell Labs and Agere in USA. His research interest includes; ultra high speed based DWDM systems transmission, optical soliton, OCDM, OTDM and network design and development. His research has led to numerous record-breaking ultra high capacity (multi-terra bits) Metro, Ultra Long Haul and Submarine system experiments. Dr. Gupta received best paper in Optical Fiber Communication Conference in 2002 by breaking both multi-terra bits capacity and ultra long distance transmission barrier, selected by IEEE/OSA technical program committee. Dr. Gupta has authored over 60 journal and conference publications and has been an invited speaker on optical fiber technologies and optical networking at premier communications conferences around the world. $\mathrm{He}$ received his B.E. in Electronics \& Telecommunications from Rani Durgavati University, Jabalpur, India and his Ph.D. Degrees in Electronics \& Electrical Engineering from the University of Strathclyde, Glasgow, UK. 